\title{
Gaussian Approximate Message Passing Detection of Orthogonal Time Frequency Space Modulation
}

\author{
Luping Xiang, Member, IEEE, Yusha Liu, Lie-Liang Yang, Fellow, IEEE and Lajos Hanzo, Fellow, IEEE
}

\begin{abstract}
Message passing (MP) aided detectors are designed for orthogonal time frequency space (OTFS) modulated system. We first characterize the transmit-receive relationship of our OTFS-modulated system in the delay-Doppler (DD) domain by designing a joint factor graph. Based on this joint factor graph, we then conceive a joint message passing aided (JMPA) detector. Furthermore, a novel low-complexity Gaussian approximate MP (GA-MP) detector is also conceived, which models the a posteriori probabilities propagated through the proposed joint factor graph by Gaussian distributions. Finally, we demonstrate that the proposed GA-MP detector outperforms the state-of-the-art MP detector by at least $1.5 \mathrm{~dB}$ at a bit error ratio (BER) of $10^{-4}$, while keeping the same complexity order.
\end{abstract}

OTFS modulation, message passing, Gaussian approximation, Doppler frequency, time-varying channel.

\section{INTRODUCTION}

Orthogonal frequency division multiplexing (OFDM) standardized in the current 3GPP Release 16 suffers from significant performance loss when the Doppler spread is relatively high, as in high-velocity railway, unmanned aerial vehicle (UAV) or aeronautical communications [1]. As a remedy, orthogonal time frequency space (OTFS) modulation has been proposed [2-4], which employs the symplectic finite Fourier transform (SFFT) before the classic discrete Fourier transform (DFT) of OFDM modulation, for transmission over multi-path delay-Doppler (DD) channels.

Optimal OTFS detection can be achieved by the maximum a posteriori (MAP) algorithm at the cost of exponentially increasing complexity. For mitigating this potentially excessive complexity, Li et al. [5] designed an attractive hybrid OTFS receiver integrating parallel interference cancellation (PIC) and MAP detection, while low-complexity message passing (MP) detection was proposed in [3], which models the inter-symbol interference (ISI) by a Gaussian distribution. However, this coarse model resulted in a degraded bit error ratio (BER), compared to that of the MAP detection. A whole suite of sub-optimal detectors has been proposed in [6-10], which demonstrate different performance vs. complexity trade-offs. For example, Yuan et al. [9] improved the convergence rate of the MP detector by proposing a variational Bayes (VB) technique, whereas Yuan et al. [10] adopted a unitary transformation for diagonalizing the system's transfer matrix, hence improving the BER performance. However, due to the sparsity existing in the DD domain, the number of interfering symbols

The authors are with School of Electronics and Computer Science, University of Southampton, SO17 1BJ, UK. (E-mail: 1x2e19, yl6g15, 1ly, lh@soton.ac.uk).

L. Hanzo would like to gratefully acknowledge the financial support of the Engineering and Physical Sciences Research Council projects EP/Noo4558/1, EP/PO34284/1, COALESCE, of the Royal Society's Global Challenges Research Fund Grant as well as of the European Research Council's Advanced Fellow Grant QuantCom (Grant No. 789028).
TABLE I

CONTRASTING THE CONTRIBUTIONS OF THIS WORK TO THE LITERATURE.

\begin{tabular}{l|c|c|c|c|c|c}
\hline Contributions & this work & {$[3]$} & {$[5]$} & {$[8]$} & {$[9]$} & {$[10]$} \\
\hline \hline Factor graph design & $\checkmark$ & $\checkmark$ & & & & \\
\hline JMPA detection & $\checkmark$ & & & & & \\
\hline Gaussian approximation & $\checkmark$ & $\checkmark$ & & $\checkmark$ & $\checkmark$ & $\checkmark$ \\
\hline Interference cancellation & $\checkmark$ & $\checkmark$ & $\checkmark$ & & & \\
\hline
\end{tabular}

for a certain symbol is typically low $[2,3]$, which leads to a performance degradation when employing the MP detector, because the central-limit theorem-based Gaussian interference assumption is rather inaccurate $[3,9,10]$.

Against this backdrop, we propose a novel Gaussian approximate MP (GA-MP) detection by modeling the individual transmit signals by Gaussian distributions, rather than approximating the ISI. The novelty of this contribution is boldly and explicitly contrasted to the state-of-the-art in Table I at a glance and the main contributions of this letter are further detailed, as follows.

- We first characterize the transmit-receive relationship of the OTFS system in the DD domain by proposing a joint factor graph, comprising all transmit symbols generated in the DD domain, which will be transmitted by $M$ subcarriers over $N$ time slots, as variable nodes (VNs) and all received symbols in the DD domain as check nodes (CNs). Based on the joint factor graph, we apply the joint message passing aided (JMPA) detection for our OTFS-modulated system.

- A novel low-complexity GA-MP detector relying on Gaussian approximation is proposed in this letter, which models the a posteriori probabilities of each transmit symbol as a Gaussian distribution. This is in contrast to the MP detection originally proposed in [3], which approximates the ISI by a Gaussian random variable.

- We benchmark the proposed GA-MP detector both against the JMPA and other low-complexity MP-based detectors found in the open literature by Monte-Carlo simulations, demonstrating that at least $1.5 \mathrm{~dB}$ gain is attained by the proposed GA-MP detector over the stateof-the-art low-complexity detectors at the BER of $10^{-4}$.

The rest of this letter is structured as follows. Section II reviews the system model of the OTFS-modulated system, while Section III details our proposed GA-MP detector. Following this, Section IV characterises the BER performance and computational complexity of the JMPA and GA-MP detectors for the OTFS-modulated system. Finally, main conclusions of this letter is summarised in Section V. 


\section{SySTEM MODEL}

In this section, we review the transmitter and receiver model of an OTFS-modulated system, along with the assumptions of this letter.

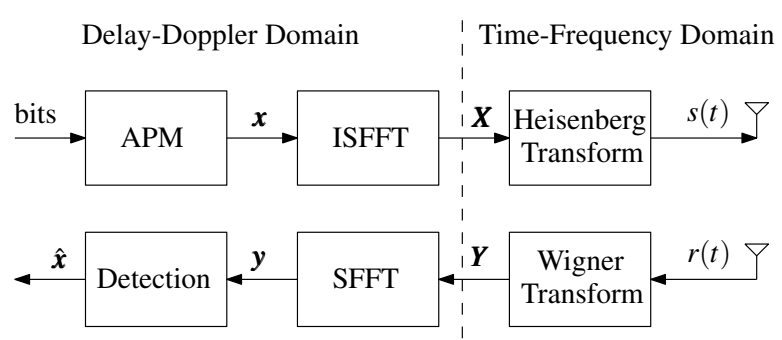

Fig. 1. Orthogonal time frequency space (OTFS)-modulated system.

When considering an end-to-end communication system employing OTFS modulation, as shown in Fig. 1, we first employ the $S$-ary amplitude phase modulation (APM) at the transmitter to map the information bits generated in the DD domain to the APM symbol $x[k, l] \in \mathcal{S}$, where $k=0,1, \cdots, N-1, l=0,1, \cdots, M-1$ are the Doppler shift index and delay index in the DD domain, respectively, and $\mathcal{S}$ is the APM symbol set. Then the inverse SFFT (ISFFT) converts the DD domain APM symbol $x[k, l]$ to the timefrequency (TF) domain, which can be expressed as

$$
\begin{aligned}
X[n, m]= & \frac{1}{N M} \sum_{k=0}^{N-1} \sum_{l=0}^{M-1} x[k, l] \exp \left[j 2 \pi\left(\frac{n k}{N}-\frac{m l}{M}\right)\right], \\
& n=0, \cdots, N-1, m=0, \cdots, M-1 .
\end{aligned}
$$

Then the Heisenberg transform ${ }^{1}$ converts the signal to the continuous waveform, giving the transmit signal $s(t)$, formulated as

$$
s(t)=\sum_{n=0}^{N-1} \sum_{m=0}^{M-1} X[n, m] \exp [j 2 \pi m \Delta f(t-n T)] g_{t x}(t-n T),
$$

where $g_{t x}(t)$ is the pulse shaping filter, $\Delta f$ is the subcarrier spacing and $T$ is the symbol duration.

In this letter, we assume that the channel experiences timevarying Rayleigh fading having $L$ paths, which has a channel impulse response (CIR) of

$$
h(\tau, \nu)=\sum_{\ell=1}^{L} \bar{h}_{\ell} \delta\left(\tau-\tau_{\ell}\right) \delta\left(\nu-\nu_{\ell}\right)
$$

where $\tau_{\ell}$ and $\nu_{\ell}$ are the delay and Doppler shift associated with the $\ell$-th path, respectively. Following the conventionally used notations, we define $\tau_{\ell}=l_{\tau_{\ell}} \frac{1}{M \Delta f}$ and $\nu_{\ell}=k_{\nu_{\ell}} \frac{1}{N T}$, where the integers $l_{\tau_{\ell}}$ and $k_{\nu_{\ell}}$ are the delay and Doppler index associated with the $\ell$-th path, respectively. Additionally, $\bar{h}_{\ell}$ follows the Gaussian distribution $\mathcal{N}_{\mathbb{C}}(0,1 / L)$ with a mean of zero and a variance of $1 / L$. Then the continuous received signal is given by

$$
r(t)=\iint h(\tau, \nu) \exp [j 2 \pi \nu(t-\tau)] s(t-\tau) d \tau d \nu+z(t),
$$

where $z(t)$ is the complex additive white Gaussian noise (AWGN) with a mean of zero and a variance of $\sigma^{2}$.

\footnotetext{
${ }^{1}$ Heisenberg transform is the general form of the IFFT for OFDM [3].
}

At the receiver, we adopt $g_{r x}(t)$ as the receive filter and transform the received signal to the TF domain by the Wigner transform ${ }^{2}$. By sampling at $t=n T$ and using $f=m \Delta f$, the received samples are given by

$$
Y[n, m]=\int r(t) g_{r x}(t-n T) \exp [-j 2 \pi m \Delta f(t-n T)] d t .
$$

The corresponding DD domain signal is obtained after the SFFT, as shown in Fig. 1, which can be expressed as

$$
y[k, l]=\frac{1}{N M} \sum_{n=0}^{N-1} \sum_{m=0}^{M-1} Y[n, m] \exp \left[-j 2 \pi\left(\frac{n k}{N}-\frac{m l}{M}\right)\right] .
$$

Under the assumption of ideal pulse shaping [11], the vectorial form of the received signal $\boldsymbol{y}=[y[0,0], \cdots, y[0, M-$ $1], \cdots, y[N-1, M-1]]^{T}$ is formulated as

$$
\boldsymbol{y}=\overline{\boldsymbol{H}} \boldsymbol{x}+\boldsymbol{z},
$$

where $\boldsymbol{x}=[x[0,0], \cdots, x[0, M-1], \cdots, x[N-1, M-1]]^{T}$ and $\overline{\boldsymbol{H}}=\sum_{\ell=1}^{L} \bar{h}_{\ell} \boldsymbol{T}^{(\ell)}$ is the $(N M \times N M)$-dimensional effective channel matrix in the DD domain, where the element $T_{p, q}^{(\ell)}$ in the $p$-th row and $q$-th column of $\boldsymbol{T}^{(\ell)}$ is given in (8), where $n=\left\lfloor\frac{p}{M}\right\rfloor$ and $m=p-n M$. This also indicates that each row and column of $\overline{\boldsymbol{H}}$ only has $L$ non-zero values. An example of $\overline{\boldsymbol{H}}$ for 3 DD taps of $(0,0),(1,1),(0,2)$ can be expressed in (9).

\section{Detection Algorithms}

In this section, we detail the optimal MAP detection in Section III-A. Following this, we design our joint factor graph representation for the OTFS system and propose the lowcomplexity GA-MP detector in Section III-B.

\section{A. Maximum a posteriori Detection}

Given the received signal $\boldsymbol{y}$ and $\overline{\boldsymbol{H}}$ of (9) estimated at the receiver, the optimum MAP detector finds $\hat{x}$ that maximizes the $a$ posteriori probability of the transmitted symbol $x$, i.e., $\operatorname{Pr}(\boldsymbol{x} \mid \boldsymbol{y})$ by the full search process, which can be formulated as

$$
\hat{\boldsymbol{x}}=\arg \max _{\boldsymbol{x} \in \mathcal{S}^{N M}} \operatorname{Pr}(\boldsymbol{x} \mid \boldsymbol{y}) .
$$

The $u$-th element $x_{u}, 0 \leq u \leq N M-1$ of $x$ may be estimated by maximizing its a posteriori probability mass function (PMF). By calculating the marginal of the a posteriori PMF function given by (10) with respect to $x_{u}, 0 \leq u \leq N M-1$, we have

$$
\hat{x}_{u}=\arg \max _{x_{u} \in \mathcal{S}} \sum_{\boldsymbol{x} \in \mathcal{S}^{N M-1}} \operatorname{Pr}(\boldsymbol{x} \mid \boldsymbol{y}),
$$

According to Bayes' rule, we have

$$
\operatorname{Pr}(\boldsymbol{x} \mid \boldsymbol{y})=\frac{\operatorname{Pr}(\boldsymbol{y} \mid \boldsymbol{x}) \operatorname{Pr}(\boldsymbol{x})}{\operatorname{Pr}(\boldsymbol{y})} \propto \operatorname{Pr}(\boldsymbol{y} \mid \boldsymbol{x}) \operatorname{Pr}(\boldsymbol{x}) .
$$

where $\operatorname{Pr}(\boldsymbol{x})=\prod_{u=0}^{N M-1} \operatorname{Pr}\left(x_{u}\right)$ is the joint a priori PMF of all the symbols in the DD domain under the assumption that the transmit signal $\boldsymbol{x}$ is independent and identically distributed

\footnotetext{
${ }^{2}$ Similar to the Heisenberg transform, the Wigner transform is the general form of the FFT for OFDM [3]
} 


$$
T_{p, q}^{(\ell)}=\left\{\begin{array}{cc}
\exp \left[-j 2 \pi\left(\frac{n}{N}-\frac{k_{\nu_{\ell}}\left(\left[m-l_{\tau_{\ell}}\right]_{M}\right)}{N M}\right)\right], & \text { if } q=\left[m-l_{\tau_{\ell}}\right]_{M}+M\left[n-k_{\nu_{\ell}}\right]_{N} \text { and } m<l_{\tau_{\ell}} \\
\exp \left[j 2 \pi \frac{k_{\nu_{\ell}}\left(\left[m-l_{\tau_{\ell}}\right]_{M}\right)}{N M}\right], & \text { if } q=\left[m-l_{\tau_{\ell}}\right]_{M}+M\left[n-k_{\nu_{\ell}}\right]_{N} \text { and } m \geq l_{\tau_{\ell}} \\
0, & \text { otherwise }
\end{array}\right.
$$

$$
\overline{\boldsymbol{H}}=\left[\begin{array}{cccccc}
\bar{h}_{1} & 0 & \bar{h}_{3} & 0 & 0 & \bar{h}_{2} \exp \left(-j 2 \pi \frac{2}{3}\right) \\
0 & \bar{h}_{1} & 0 & \bar{h}_{3} \exp \left(-j 2 \pi \frac{1}{2}\right) & \bar{h}_{2} \exp \left(-j 2 \pi \frac{1}{6}\right) & 0 \\
0 & \bar{h}_{2} & \bar{h}_{1} & 0 & \bar{h}_{3} & 0 \\
\bar{h}_{2} & 0 & 0 & \bar{h}_{1} & 0 & \bar{h}_{3} \exp \left(-j 2 \pi \frac{1}{2}\right) \\
\bar{h}_{3} & 0 & 0 & \bar{h}_{2} \exp \left(-j 2 \pi \frac{5}{6}\right) & \bar{h}_{1} & 0 \\
0 & \bar{h}_{3} & \bar{h}_{2} \exp \left(-j 2 \pi \frac{5}{6}\right) & 0 & 0 & \bar{h}_{1}
\end{array}\right]
$$

(i.i.d.) and $\operatorname{Pr}(\boldsymbol{y})=\sum_{\boldsymbol{x} \in \mathcal{S}^{N M}} \operatorname{Pr}(\boldsymbol{y} \mid \boldsymbol{x}) \operatorname{Pr}(\boldsymbol{x})$ is the probability density function (PDF) of the received signal $y$.

Furthermore, since the noise vector $n$ is assumed to be i.i.d. as well, we have

$$
\operatorname{Pr}(\boldsymbol{y} \mid \boldsymbol{x})=\prod_{j=0}^{N M-1} \operatorname{Pr}\left(y_{j} \mid \boldsymbol{x}\right)
$$

where $\operatorname{Pr}\left(y_{j} \mid x\right), 0 \leq j \leq N M-1$ is the conditional probability of the $j$-th observation $y_{j}$, given the transmitted signal $\boldsymbol{x}$. Additionally, following the expression of $\overline{\boldsymbol{H}}$ in (9), we can see that each symbol has only $(L-1)(L \ll N M)$ interfering symbols. Hence, by exploiting the sparsity of $\overline{\boldsymbol{H}}$ in (13) we arrive at

$$
\operatorname{Pr}\left(y_{j} \mid \boldsymbol{x}\right)=\operatorname{Pr}\left(y_{j} \mid \boldsymbol{x}_{[j]}\right),
$$

where $[j]$ represents the column index set of the $L$ non-zero elements in the $j$-th row of $\bar{H}$. Furthermore, by combining (12), (13), and (14) into (11) and removing all unnecessary constant scalars, we arrive at:

$$
\hat{x}_{u}=\arg \max _{x_{u} \in \mathcal{S}} \sum_{\boldsymbol{x} \in \mathcal{S}^{L(L-1)}, x_{u}} \operatorname{Pr}(\boldsymbol{x}) \prod_{j \in \mathcal{V}_{u}} \operatorname{Pr}\left(y_{j} \mid \boldsymbol{x}_{[j]}\right),
$$

where $\mathcal{V}_{u}$ is the non-zero index set for the $u$-th column of $\overline{\boldsymbol{H}}$ and $\operatorname{Pr}\left(y_{j} \mid \boldsymbol{x}_{[j]}\right)$ is formulated as

$$
\operatorname{Pr}\left(y_{j} \mid \boldsymbol{x}_{[j]}\right)=\frac{1}{\sqrt{2 \pi} \sigma} \exp \left(-\frac{1}{2 \sigma^{2}}\left|y_{j}-\overline{\boldsymbol{H}}_{[j]} \boldsymbol{x}_{[j]}\right|^{2}\right) .
$$

Observe from (15) that the detection complexity of this MAP algorithm is $\mathcal{O}\left(|\mathcal{S}|^{L(L-1)} N M\right)$, which is unaffordable for large-scale OTFS-modulated systems. Hence, low-complexity detection is required for practical system implementations.

\section{B. Gaussian Approximate Message Passing (GA-MP) Detec- tion}

Before we discuss our GA-MP detection, we propose our joint factor graph design tailored for the OTFS system. In contrast to the factor graph that was used for representing mmWave channels in the TF domain $[12,13]$, we illustrate the input-output relationship of the channel in the DD domain. We commence by mapping our $u$-th non-zero DD-domain signal $x_{u}$ to the $u$-th variable node (VN). Similarly, the received signal $y_{j}$ can be treated as the $j$-th check node (CN). Considering the example of $\overline{\boldsymbol{H}}$ in (9), the factor graph representing the connections between the received signal $y$ and the DD-domain transmitted signal $x$ is demonstrated in Fig.
2, where we have $M N=6$ and $L=3$. In this example, the connections between the VNs and CNs indicate the positions of non-zero elements in (9). For example, the 1-st VN in Fig. 2 is connected to the 1-st, 4-th and 5-th CNs, which corresponds to the non-zero positions of the first column in the matrix $\bar{H}$ given by (9).

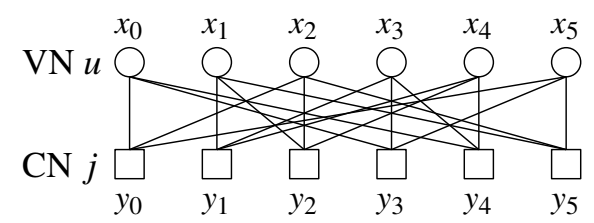

Fig. 2. An example of joint factor graph representation as (9) of an OTFS system with $M N=6$ and $L=3$.

Based on the joint factor graph, we can simultaneously calculate the messages of all $x$ exchanged via the edges of the factor graph, rather than modelling the interference imposed by other symbols as a Gaussian random variable, which is referred to as the MP detection in [3,6].

As shown in Fig. 2, each $\mathrm{VN} u / \mathrm{CN} j$ is connected to $L \mathrm{CNs} / \mathrm{VNs}$. Hence, we can define an index set $\mathcal{V}_{u}$ comprising the CNs that have connections to $\mathrm{VN} u$ as $\mathcal{V}_{u}=\left\{j: 0 \leqslant j \leqslant N M-1, \bar{H}_{j, u} \neq 0\right\}$. Similarly, an index set $\mathcal{C}_{j}$ representing the connections to $\mathrm{CN} j$ can be defined as $\mathcal{C}_{j}=\left\{u: 0 \leqslant u \leqslant N M-1, \bar{H}_{j, u} \neq 0,\right\}$.

Following the factor graph and the principles of the sumproduct algorithm $[14,15]$, the information transmitted from $\mathrm{CN} j$ to $\mathrm{VN} u$ is the sum of all CNs that have connections to $\mathrm{VN} u$, which can be expressed as

$$
\begin{aligned}
\delta_{j \rightarrow u}^{\left(x_{u}, i\right)}= & \sum_{\boldsymbol{x}_{[j]} \in \mathcal{S}^{L-1}, x_{u}}\left(\prod_{x_{u}^{\prime} \in \boldsymbol{x}_{[j]} \backslash x_{u}} \eta_{u \rightarrow j}^{\left(x_{u}^{\prime}, i-1\right)}\right) \operatorname{Pr}\left(y_{j} \mid \boldsymbol{x}_{[j]}\right), \\
& j=0,1, \cdots, N M-1, u=0,1, \cdots, N M-1,
\end{aligned}
$$

where $\operatorname{Pr}\left(y_{j} \mid x_{[j]}\right)$ is given in (16). By contrast, the information conveyed from $\mathrm{VN} u$ to $\mathrm{CN} j$ is the product of the information collected from the VNs connected to $\mathrm{CN} j$ excluding $\mathrm{VN} u$, which can be expressed as

$$
\begin{aligned}
\eta_{u \rightarrow j}^{\left(x_{u}, i\right)}= & \varepsilon_{u \rightarrow j} \prod_{j^{\prime} \in \mathcal{V}_{u} \backslash j} \delta_{j^{\prime} \rightarrow u}^{\left(x_{u}, i\right)}, \\
& j=0,1, \cdots, N M-1, u=0,1, \cdots, N M-1 .
\end{aligned}
$$

Observe from (17) that the complexity order of JMPA detection is $\mathcal{O}\left(|\mathcal{S}|^{L} N M\right)$, which increases exponentially with the 
increase of $L$. Hence, with the objective of further reducing the detection complexity, we approximate the downward oriented information $\eta_{u \rightarrow j}^{\left(x_{u}, i-1\right)}$ transmitted from the $\mathrm{VN} u$ to the $\mathrm{CN} j$ in the $(i-1)$-th iteration by the Gaussian function $\hat{\eta}_{u \rightarrow j}^{\left(x_{u}, i-1\right)}$, which can be expressed as

$$
\eta_{u \rightarrow j}^{\left(x_{u}, i-1\right)}=\mathcal{N}_{\mathbb{C}}\left(x_{u} ; \mu_{u \rightarrow j}^{(i-1)}, \sigma_{u \rightarrow j}^{(i-1)}\right),
$$

where $\mu_{u \rightarrow j}^{(i-1)}$ and $\sigma_{u \rightarrow j}^{(i-1)}$ are the mean and variance of the Gaussian variable $x_{u}$. The corresponding expression of $\mu_{u \rightarrow j}^{(i-1)}$ and $\sigma_{u \rightarrow j}^{(i-1)}$ will be detailed later in this section.

Then, the upward-oriented information $\delta_{j \rightarrow u}^{\left(x_{u}, i\right)}$ conveyed from the $\mathrm{CN} j$ to the $\mathrm{VN} u$ in the $i$-th iteration can also be approximated by a Gaussian function expressed as

$$
\delta_{j \rightarrow u}^{\left(x_{u}, i\right)}=\mathcal{N}_{\mathbb{C}}\left(\bar{H}_{j, u} x_{u} ; \alpha_{j \rightarrow u}^{(i)}, \beta_{j \rightarrow u}^{(i)}\right),
$$

where the mean is $\alpha_{j \rightarrow u}^{(i)}=y_{j}-\sum_{u^{\prime} \in \mathcal{C}_{j} \backslash u}\left(\bar{H}_{j, u^{\prime}} \mu_{u^{\prime} \rightarrow j}^{(i)}\right)$ and the variance is $\beta_{j \rightarrow u}^{(i)}=\sigma^{2}+\sum_{u^{\prime} \in \mathcal{C}_{j} \backslash u}\left|\bar{H}_{j, u^{\prime}}\right|^{2} \sigma_{u^{\prime} \rightarrow j}^{(i)}$ [16].

Later, $\eta_{u \rightarrow j}^{\left(x_{u}, i\right)}$ is updated by the values $\delta_{j \rightarrow u}^{\left(x_{u}, i\right)}$ obtained in the $i$-th inner iteration as follows

$$
\eta_{u \rightarrow j}^{\left(x_{u}, i\right)}=\varepsilon_{u \rightarrow j} \prod_{j^{\prime} \in \mathcal{V}_{u} \backslash j} \delta_{j^{\prime} \rightarrow u}^{\left(x_{u}, i\right)},
$$

where $\varepsilon_{u \rightarrow j}$ is the normalisation factor ensuring that $\sum_{x_{u} \in \mathcal{S}} \eta_{u \rightarrow j}^{\left(x_{u}, i\right)}=1$.

Upon substituting (20) into (21), we have

$$
\eta_{u \rightarrow j}^{\left(x_{u}, i\right)}=\frac{\mathcal{N}_{\mathbb{C}}\left(x_{u} ; \xi_{u \rightarrow j}^{(i)}, \gamma_{u \rightarrow j}^{(i)}\right)}{\sum_{x_{u} \in \mathcal{S}} \mathcal{N}_{\mathbb{C}}\left(x_{u} ; \xi_{u \rightarrow j}^{(i)}, \gamma_{u \rightarrow j}^{(i)}\right)},
$$

where

$$
\begin{gathered}
\xi_{u \rightarrow j}^{(i)}=\gamma_{u \rightarrow j}^{(i)} \sum_{j^{\prime} \in \mathcal{V}_{u} \backslash j} \frac{\bar{H}_{j^{\prime}, u}^{*} \alpha_{j^{\prime} \rightarrow u}^{(i)}}{\beta_{j^{\prime} \rightarrow u}^{(i)}}, \\
\gamma_{u \rightarrow j}^{(i)}=\left(\sum_{j^{\prime} \in \mathcal{V}_{u} \backslash j} \frac{\left|\bar{H}_{j^{\prime}, u}\right|^{2}}{\beta_{j^{\prime} \rightarrow u}^{(i)}}\right)^{-1} .
\end{gathered}
$$

Additionally, for improving the overall detection performance by controlling the convergence rate, we apply a damping factor $d(d \in(0,1])$ before proceeding to the next iteration, which can be expressed as

$$
\bar{\eta}_{u \rightarrow j}^{\left(x_{u}, i\right)}=d \cdot \eta_{u \rightarrow j}^{\left(x_{u}, i\right)}+(1-d) \cdot \bar{\eta}_{u \rightarrow j}^{\left(x_{u}, i-1\right)} .
$$

Let us now discuss the expressions of $\eta_{u \rightarrow j}^{\left(x_{u}, i\right)}$. A popular technique of finding the GA of $\eta_{u \rightarrow j}^{\left(x_{u}, i\right)}$ is to minimize the inclusive Kullback-Leibler (KL) divergence of $\operatorname{KL}\left(\eta_{u \rightarrow j}^{\left(x_{u}, i\right)} \| \hat{\eta}_{u \rightarrow j}^{\left(x_{u}, i\right)}\right)[9,17]$, which gives the expression of $\mu_{u \rightarrow j}^{(i)}$ and $\sigma_{u \rightarrow j}^{(i)}$ as

$$
\begin{gathered}
\mu_{u \rightarrow j}^{(i)}=\sum_{x_{u} \in \mathcal{S}} x_{u} \cdot \bar{\eta}_{u \rightarrow j}^{\left(x_{u}, i\right)}, \\
\sigma_{u \rightarrow j}^{(i)}=\sum_{x_{u} \in \mathcal{S}}\left|x_{u}\right|^{2} \bar{\eta}_{u \rightarrow j}^{\left(x_{u}, i\right)}-\left|\mu_{u \rightarrow j}^{(i)}\right|^{2} .
\end{gathered}
$$

Furthermore, the a posteriori probability $\eta_{u}^{\left(x_{u}, i\right)}$ before damping is expressed as

$$
\eta_{u}^{\left(x_{u}, i\right)}=\frac{\mathcal{N}_{\mathbb{C}}\left(x_{u} ; \xi_{u}^{(i)}, \gamma_{u}^{(i)}\right)}{\sum_{x_{u} \in \mathcal{S}} \mathcal{N}_{\mathbb{C}}\left(x_{u} ; \xi_{u}^{(i)}, \gamma_{u}^{(i)}\right)},
$$

where $\xi_{u}^{(i)}=\gamma_{u}^{(i)} \sum_{j \in \mathcal{V}_{u}} \frac{\bar{H}_{j, u}^{*} \alpha_{j \rightarrow u}^{(i)}}{\beta_{j \rightarrow u}^{(i)}}$ and $\gamma_{u}^{(i)}=$ $\left(\sum_{j \in \mathcal{V}_{u}} \frac{\left|\bar{H}_{j, u}\right|^{2}}{\beta_{j \rightarrow u}^{(i)}}\right)^{-1}$.

In order to mitigate the ISI and reduce the detection complexity, we set a threshold $\epsilon \in(0,1]$ for $\bar{\eta}_{u}^{\left(x_{u}, i\right)}$. When $\bar{\eta}_{u}^{\left(x_{u}, i\right)}>\epsilon$, we stop updating $\bar{\eta}_{u}^{\left(x_{u}, i\right)}$ in the following iterations and treat the corresponding detected $\hat{x}_{u}^{(i)}$ as the final detection result. By contrast, a lower $\epsilon$ may significantly reduce the complexity but it also leads to error propagation. Based on our experiments, we select $\epsilon=0.99$ in our simulations, which shows a good BER vs. complexity trade-off.

Now, based on (19) to (28), we observe that the complexity order of the GA-MP detection becomes $\mathcal{O}(|\mathcal{S}| L N M)$.

\section{RESULTS}

In this section, we characterize the BER performance and computational complexity of our proposed GA-MP detector under different system set-ups in Sections IV-A and IV-B, respectively, using the parameters of Table II.

TABLE II

SIMULATION PARAMETERS

\begin{tabular}{l|r}
\hline Parameters & Values \\
\hline Carrier frequency & $4 \mathrm{GHz}$ \\
\hline No. of subcarriers $(M)$ & 16 \\
\hline No. of OTFS symbols $(N)$ & 8 \\
\hline Maximum delay index $\left(l_{\tau_{\max }}\right)$ & 3 \\
\hline Doppler index range $\left(\left[-k_{\nu_{\max }}, k_{\nu_{\max }}\right]\right)$ & {$[-3,3]$} \\
\hline Velocity & $150,300 \mathrm{Kmph}$ \\
\hline No. of Paths $(L)$ & 4,8 \\
\hline Damping factor $(d)$ & 0.5 \\
\hline No. of iterations $(I)$ & 10 \\
\hline
\end{tabular}

Specifically, we consider an OTFS-modulated system operating at $4 \mathrm{GHz}$ frequency band and $M=16$ subcarriers are employed for all simulations in this section. Since the focus of this short letter is on detector design, we assume that the channel state information (CSI) has been perfectly estimated at the receiver. The channel estimation techniques that have been proposed for OFDM systems relying on signal processing in the TF domain can also be readily extended for estimating the DD-domain channel for OTFS system $[18,19]$. Additionally, the Doppler index of the $\ell$-th path is generated with equal probabilities from the set $\left[-k_{\nu_{\max }}, k_{\nu_{\max }}\right]$, while the delay index set is $\left[1, l_{\tau_{\max }}\right]$ and $l_{\tau_{1}}=0$. The damping factor of $d=0.5$ is selected by trivial experiments and is employed for all MP-based detectors considered in this work. The number of iterations employed for all MP-based detectors is fixed at $I=10$.

\section{A. BER Performance}

We commence by comparing the BER performance of our proposed GA-MP to that of various low-complexity state-ofthe-art OTFS detectors. Specifically, Fig. 3 shows the BER 


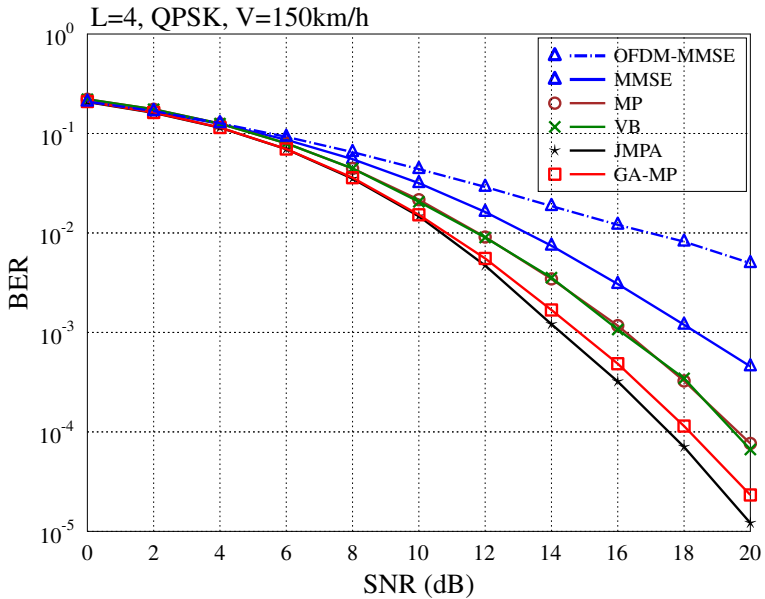

Fig. 3. BER comparison of the MMSE [7], MP [3], VB-MP [9], JMPA and the proposed GA-MP detection for the OTFS-modulated system communicating over time-varying Rayleigh fading channels having $L=4$ paths in the DD domain, where the mobile speed is $150 \mathrm{Kmph}$.

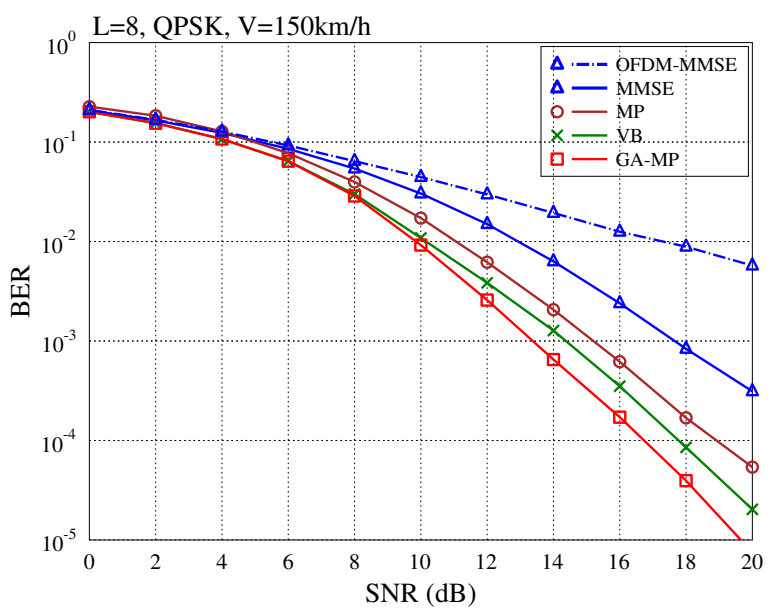

Fig. 4. BER comparison of the MMSE [7], MP [3], VB-MP [9] and the proposed GA-MP detection for the OTFS-modulated system communicating over time-varying Rayleigh fading channels having $L=8$ paths in the DD domain, where the mobile speed is $150 \mathrm{Kmph}$.

performance of the MMSE [7], MP [3], VB-MP [9], JMPA and the proposed OTFS GA-MP detectors for $L=4$ paths in the TF domain. Furthermore, the conventional MMSE OFDM detector is also included in Fig. 3 as a benchmark. We can see that the JMPA detector achieves the best BER performance among these detectors, since this relies on the exact calculation of the a posteriori probabilities exchanged within the factor graph. Furthermore, the BER performance of our GA-MP detector approaches that of the JMPA detector and outperforms all the other state-of-the-art low-complexity detectors.

Furthermore, when the number of paths $L$ increases from 4 to 8 , the complexity of the JMPA detector, which increases exponentially with $L$, becomes unaffordable. By contrast, the low-complexity detectors including the MMSE [7], MP [3], VB-MP [9] and our proposed GA-MP detector can reliably detect the received OTFS signal. Hence, Fig. 4 characterizes the BER performance of the above-mentioned detectors, among which our proposed GA-MP detector still achieves the best BER performance.

By comparing Figs. 3 and 4, we can observe a $1.5 \mathrm{~dB}$ SNR gain at the BER of $10^{-4}$ when the number of channel paths

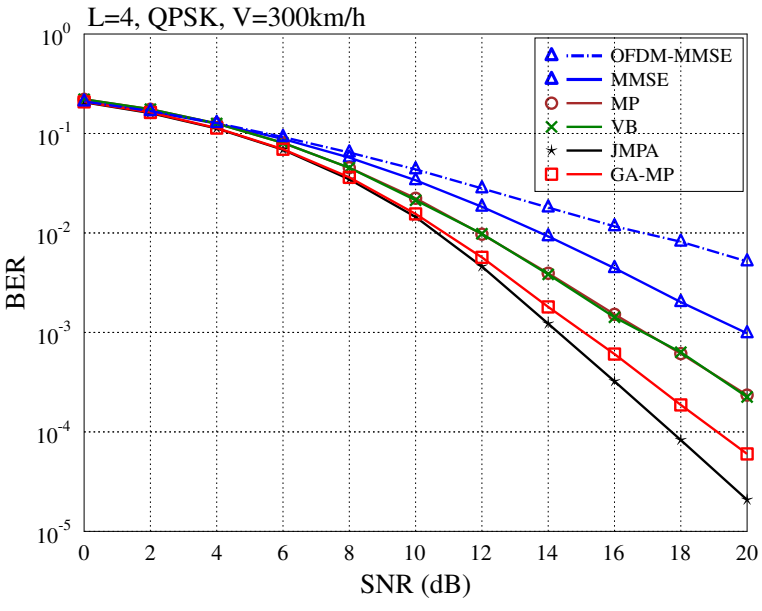

Fig. 5. BER comparison of the MMSE [7], MP [3], VB-MP [9], JMPA and the proposed GA-MP detection for the OTFS-modulated system communicating over time-varying Rayleigh fading channels having $L=4$ paths in the DD domain, where the mobile speed is $300 \mathrm{Kmph}$.

in the DD domain increases from $L=4$ to 8 and when the GA-MP detection is employed. This is because a higher $L$ leads to a denser factor graph, hence the decision of each $\mathrm{VN}$ is determined by a higher number of $\mathrm{CNs}$ of the factor graph. Hence, a higher $L$ in the DD domain also enhances the system's BER performance, albeit at the cost of a higher detection complexity.

Finally, Fig. 5 investigates the BER performance of the OTFS system employing above-mentioned detectors a higher mobile speed of $300 \mathrm{Kmph}$, with the rest parameters being the same as in Fig. 3. By comparing Fig. 5 to Fig. 3, we can see a slight performance degradation for the higher mobile speed of $300 \mathrm{Kmph}$, due to higher Doppler frequency. For example, when the proposed GA-MP detector is employed, only less than $1 \mathrm{~dB}$ erosion is observed, showing a promising potential of high-speed communication scenarios in the next generation communication networks, such as air-to-air and air-to-space communications.

\section{B. Complexity}

Now we compare the complexity of the MP-based detectors mentioned above in terms of the number of floating point operations (FLOPs) required per iteration. The computational complexity of the JMPA detector is mainly determined by the VN update of (17) and $\mathrm{CN}$ update of (18). Following the analysis of [20], the number of FLOPs required to perform JMPA detection in each iteration is expressed as

$$
\mathcal{C}_{\text {JMPA }}=N M L\left[(7 L-1)|\mathcal{S}|^{L}+(L-1)|\mathcal{S}|\right] .
$$

We can see that the increase of $L$ will lead to an exponential escalation of $\mathcal{C}_{\mathrm{JMPA}}$. By contrast, the number of FLOPs required by the MP detector of [3] per iteration is expressed as

$$
\mathcal{C}_{\mathrm{MP}}=27|\mathcal{S}| N M L+4 M N L .
$$

Note that we did not consider the employment of the threshold $\epsilon$ and the damping factor $d$ for focusing on the dominant steps of the detection process.

Finally, the detection complexity of the GA-MP detector is dominated by the computation of the VN update of (22) and 
TABLE III

COMPUTATIONAL COMPLEXITY OF JMPA, MP AND GA-MP DETECTORS.

\begin{tabular}{l||c|c|c}
\hline Detector & Complexity & Example of Fig. 3 (FLOPs) & Example of Fig. 4 (FLOPs) \\
\hline JMPA & $(29)$ & $3.55 \times 10^{6}$ & $3.69 \times 10^{9}$ \\
\hline MP & $(30)$ & $5.73 \times 10^{4}$ & $1.15 \times 10^{5}$ \\
\hline GA-MP & $(31)$ & $5.89 \times 10^{4}$ & $1.18 \times 10^{5}$ \\
\hline
\end{tabular}

CN update of (20). Hence, the number of FLOPs required for the GA-MP detector per iteration is expressed as [16]

$$
\mathcal{C}_{\mathrm{GA}-\mathrm{MP}}=23|\mathcal{S}| N M L+23 M N L \text {. }
$$

Table III shows the number of FLOPs required for the simulations of Figs. 3 and 4, where we can see that compared to the JMPA detector, both the MP detector of [3] and our proposed GA-MP detector require a much lower number of FLOPs due to the approximation of the messages propagated via the factor graph. In particular, $3.69 \times 10^{9}$ FLOPs are required for the JMPA detector to obtain the simulation results employing the parameters of Fig. 4, where $L=8$ paths are considered, which is hardly affordable for real-time implementations. Hence, low complexity detectors are required for the family OTFS systems, motivating us to propose the GA-MP detector. Additionally, while our proposed GA-MP detector achieves at least $1.5 \mathrm{~dB}$ SNR gain at the BER of $10^{-4}$, compared to the MP detector of [3], a slightly higher number of FLOPs per iteration are used.

\section{CONCLUSIONS}

The low-complexity GA-MP detector has been proposed for OTFS systems. We first characterized the transmit-receive relationship of the OTFS signal by proposing a joint factor graph and designed a JMPA detector for the OTFS system. Furthermore, the novel low-complexity GA-MP detector models the a posteriori probabilities propagated through the factor graph by the Gaussian distributions. This is in contrast to the MP detector of [3], which approximates the ISI by a Gaussian random variable. Our simulation results demonstrated at least 1.5 dB SNR gain over the state-of-the-art detection algorithms at the BER of $10^{-4}$, while maintaining the same complexity order. The promising application of the GA-MP algorithm to solve channel estimation and/or data detection problem in future wireless communication networks, such as index modulation-aided [19] and reconfigurable intelligent surface (RIS)-based systems $[21,22]$ may also be investigated in the future.

\section{REFERENCES}

[1] V. W. Wong, Key technologies for $5 G$ wireless systems. Cambridge university press, 2017.

[2] R. Hadani, S. Rakib, M. Tsatsanis, A. Monk, A. J. Goldsmith, A. F. Molisch, and R. Calderbank, "Orthogonal time frequency space modulation," in 2017 IEEE Wireless Communications and Networking Conference $(W C N C)$, pp. 1-6, IEEE, 2017.

[3] P. Raviteja, K. T. Phan, Y. Hong, and E. Viterbo, "Interference cancellation and iterative detection for orthogonal time frequency space modulation," IEEE Transactions on Wireless Communications, vol. 17, no. 10, pp. 6501-6515, 2018.

[4] P. Raviteja, K. T. Phan, and Y. Hong, "Embedded pilot-aided channel estimation for OTFS in delay-doppler channels," IEEE Transactions on Vehicular Technology, vol. 68, no. 5, pp. 4906-4917, 2019.

[5] S. Li, W. Yuan, Z. Wei, J. Yuan, B. Bai, D. W. K. Ng, and Y. Xie, "Hybrid MAP and PIC detection for OTFS modulation," arXiv preprint arXiv:2010.13030, 2020
[6] P. Raviteja, K. T. Phan, Q. Jin, Y. Hong, and E. Viterbo, "Lowcomplexity iterative detection for orthogonal time frequency space modulation," in 2018 IEEE Wireless Communications and Networking Conference (WCNC), pp. 1-6, IEEE, 2018.

[7] S. Tiwari, S. S. Das, and V. Rangamgari, "Low complexity LMMSE receiver for OTFS," IEEE Communications Letters, vol. 23, no. 12, pp. 2205-2209, 2019.

[8] L. Li, Y. Liang, P. Fan, and Y. Guan, "Low complexity detection algorithms for OTFS under rapidly time-varying channel," in 2019 IEEE 89th Vehicular Technology Conference (VTC2019-Spring), pp. 15, IEEE, 2019.

[9] W. Yuan, Z. Wei, J. Yuan, and D. W. K. Ng, "A simple variational bayes detector for orthogonal time frequency space (OTFS) modulation," IEEE Transactions on Vehicular Technology, 2020.

[10] Z. Yuan, F. Liu, W. Yuan, Q. Guo, Z. Wang, and J. Yuan, "Iterative detection for orthogonal time frequency space modulation using approximate message passing with unitary transformation," arXiv preprint arXiv:2008.06688, 2020

[11] P. Raviteja, Y. Hong, E. Viterbo, and E. Biglieri, "Practical pulseshaping waveforms for reduced-cyclic-prefix OTFS," IEEE Transactions on Vehicular Technology, vol. 68, no. 1, pp. 957-961, 2018

[12] C. Huang, L. Liu, C. Yuen, and S. Sun, "Iterative channel estimation using LSE and sparse message passing for mmwave MIMO systems," IEEE Transactions on Signal Processing, vol. 67, no. 1, pp. 245-259, 2018.

[13] C. Huang, L. Liu, C. Yuen, and S. Sun, "A LSE and sparse message passing-based channel estimation for mmwave MIMO systems," in 2016 IEEE Globecom Workshops (GC Wkshps), pp. 1-6, IEEE, 2016.

[14] F. R. Kschischang, B. J. Frey, and H.-A. Loeliger, "Factor graphs and the sum-product algorithm," IEEE Transactions on information theory, vol. 47, no. 2, pp. 498-519, 2001.

[15] R. Hoshyar, F. P. Wathan, and R. Tafazolli, "Novel low-density signature for synchronous CDMA systems over AWGN channel," IEEE Transactions on Signal Processing, vol. 56, no. 4, pp. 1616-1626, 2008.

[16] S. Wu, L. Kuang, Z. Ni, J. Lu, D. Huang, and Q. Guo, "Lowcomplexity iterative detection for large-scale multiuser MIMO-OFDM systems using approximate message passing," IEEE Journal of Selected Topics in Signal Processing, vol. 8, no. 5, pp. 902-915, 2014.

[17] W. Yuan, N. Wu, Q. Guo, Y. Li, C. Xing, and J. Kuang, "Iterative receivers for downlink MIMO-SCMA: Message passing and distributed cooperative detection," IEEE Transactions on Wireless Communications, vol. 17, no. 5, pp. 3444-3458, 2018.

[18] Q. Shi, N. Wu, X. Ma, and H. Wang, "Frequency-domain joint channel estimation and decoding for faster-than-Nyquist signaling," IEEE Transactions on Communications, vol. 66, no. 2, pp. 781-795, 2017.

[19] Q. Shi, N. Wu, H. Wang, X. Ma, and L. Hanzo, "Factor graph based message passing algorithms for joint phase-noise estimation and decoding in OFDM-IM," IEEE Transactions on Communications, vol. 68, no. 5, pp. 2906-2921, 2020.

[20] Y. Liu, L. Xiang, L.-L. Yang, and L. Hanzo, "Space-time coded generalized spatial modulation for sparse code division multiple access," IEEE Transactions on Wireless Communications, 2021

[21] C. Huang, A. Zappone, G. C. Alexandropoulos, M. Debbah, and C. Yuen, "Reconfigurable intelligent surfaces for energy efficiency in wireless communication," IEEE Transactions on Wireless Communications, vol. 18, no. 8, pp. 4157-4170, 2019

[22] C. Huang, S. Hu, G. C. Alexandropoulos, A. Zappone, C. Yuen, R. Zhang, M. Di Renzo, and M. Debbah, "Holographic MIMO surfaces for $6 \mathrm{G}$ wireless networks: Opportunities, challenges, and trends," IEEE Wireless Communications, vol. 27, no. 5, pp. 118-125, 2020. 